Rechtsmedizin 2022 · 32:249-255

https://doi.org/10.1007/s00194-021-00550-0

Angenommen: 10. November 2021

Online publiziert: 22. Dezember 2021

(c) Der/die Autor(en) 2021

\section{Rechtsmedizinische Modelle der Versorgung von gewalt- betroffenen Personen in Deutschland}

\author{
Cleo Walz ${ }^{1} \cdot$ Nadine Wilke-Schalhorst ${ }^{2} \cdot$ Clara-Sophie Schwarz $^{1} \cdot$ Tanja Germerott $^{1}$ \\ 'Institut für Rechtsmedizin, Universitätsmedizin der Johannes Gutenberg-Universität Mainz, Mainz, \\ Deutschland \\ ${ }^{2}$ Institut für Rechtsmedizin, Universitätsklinikum Schleswig-Holstein, Lübeck, Deutschland
}

Hintergrund: In Deutschland bestehen keine einheitlichen Strukturen für die Versorgung von Gewaltbetroffenen. Aktuellen politischen Bestrebungen zufolge soll eine Beweissicherung auch ohne Strafanzeige flächendeckend in Deutschland ermöglicht werden. Die Rechtsmedizin verfügt über die notwendige Expertise, um eine gerichtsverwertbare Befunderhebung niederschwellig durchzuführen und den Aufbau eines solchen Angebotes zu begleiten.

Material und Methoden: Durch die Arbeitsgemeinschaft Klinische Rechtsmedizin der Deutschen Gesellschaft für Rechtsmedizin erfolgte eine Umfrage an rechtsmedizinischen Instituten in Deutschland zu aktuellen Modellen der Versorgung von Gewaltbetroffenen. Ausgewertet wurden Angaben zur Projektstruktur, zum Leistungsangebot und zum Versorgungsgebiet, zur Finanzierung sowie zu Vor- und Nachteilen der Projekte.

Ergebnisse: Es wurden 16 Projekte aus 14 Bundesländern rückgemeldet, die körperliche Untersuchungen an eigenen und peripheren Standorten, Beratungen, Lagerungen von Asservaten sowie Fortbildungen beinhalten. In etwa der Hälfte der Projekte haben die rechtsmedizinischen Institute zusätzlich koordinierende Funktion, wobei Kooperationspartner die Untersuchungen durchführen. Der Großteil der Projekte wird über öffentliche Mittel finanziert; in 7 Projekten müssen zusätzlich eigene oder sonstige Mittel aufgebracht werden. Elf Projekte sind befristet oder Modellprojekte. Diskussion: Rechtsmedizinische Institute verfügen über die notwendige Expertise und bieten ein umfassendes niederschwelliges Angebot für Gewaltbetroffene. Die Finanzierungsmodelle sind heterogen, häufig befristet und nicht auskömmlich. Um die politisch geforderte, flächendeckende Versorgung zu etablieren, sind eine bundesweite Einbeziehung der Rechtsmedizin und auskömmliche Finanzierung dringend notwendig.

\section{Schlüsselwörter}

Klinische Rechtsmedizin · Finanzierung · Ambulanzen · Istanbul-Konvention · Masernschutzgesetz

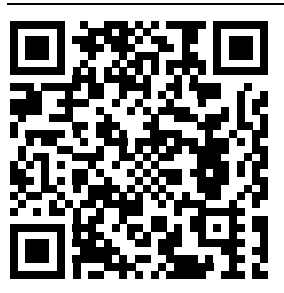

QR-Code scannen \& Beitrag online lesen

\section{Hintergrund}

Die medizinisch-forensische Versorgung von Gewaltbetroffenen ist in Deutschland nicht einheitlich geregelt. Erfolgt unmittelbar nach einem Übergriff eine Strafanzeige, wird fallabhängig eine (rechts-)medizinische Untersuchung der betroffenen Person mit nachfolgender
Gutachtenerstattung seitens der Ermittlungsbehörden beauftragt. Vor allem in Fällen häuslicher und sexualisierter Gewalt besteht jedoch bekanntermaßen eine hohe Hemmschwelle, unmittelbar nach dem Vorfall eine Strafanzeige zu erstatten. Nicht selten stellen sich Gewaltbetroffene, speziell Frauen im Kontext häuslicher oder sexualisierter Gewalt, in einer ärztli- 
chen Praxis oder in einer Klinik vor, ohne über ihre Gewalterfahrungen zu sprechen oder zu diesem Zeitpunkt eine Strafanzeige erstatten zu wollen $[2,9,13]$. Im Falle einer späteren Strafanzeige stehen dann oftmals keine objektiven und den Ansprüchen an eine gerichtsverwertbare Beweissicherung genügenden Befunde zur Verfügung [14].

Im klinischen Alltag führen unzureichende Kenntnisse über Bedeutung und Durchführung einer gerichtsverwertbaren Befundsicherung sowie das Fehlen notwendiger Materialien (z. B. spezielle Untersuchungskits) und gesicherter Lagerungsmöglichkeiten von Asservaten zu Schwierigkeiten. Aufgrund der fachlichen Ausrichtung betreffend forensische Fragestellungen und der jahrzehntelangen intensiven Zusammenarbeit mit Ermittlungsbehörden ist in der Rechtsmedizin die für eine gerichtsverwertbare Befundsicherung zwingend notwendige Expertise angesiedelt [10]. Viele rechtsmedizinische Institute haben Versorgungsstrukturen geschaffen, um in Fällen körperlicher und sexualisierter Gewalt von Erwachsenen und Kindern auch ohne Strafanzeige eine gerichtsverwertbare Befunderhebung und Spurensicherung, ggf. in Kooperation mit klinischen Ambulanzen und Kliniken, anzubieten [8].

Laut neuem Koalitionsvertrag der Bundesregierung soll eine "ressortübergreifende politische Strategie gegen Gewalt" entwickelt werden, „die Gewaltprävention und die Rechte der Betroffenen in den Mittelpunkt stellt" [6]. Zudem soll die gerichtsverwertbare vertrauliche Beweissicherung flächendeckend, wohnortnah umgesetzt werden [7]. Darüber hinaus hat der Bundestag im neuen Masernschutzgesetz beschlossen, dass künftig die sog. vertrauliche Spurensicherung bei Verdacht auf sexualisierte Gewalt von den Krankenkassen erstattet werden soll: "Zur Krankenbehandlung gehören auch Leistungen zur vertraulichen Spurensicherung am Körper, einschließlich der erforderlichen Dokumentation sowie Laboruntersuchungen und einer ordnungsgemäßen Aufbewahrung der sichergestellten Befunde, bei Hinweisen auf drittverursachte Gesundheitsschäden, die Folge einer Misshandlung, eines sexuellen Missbrauchs, eines sexuellen
Übergriffs, einer sexuellen Nötigung oder einer Vergewaltigung sein können" [3].

Die Bestrebungen resultieren möglicherweise auch aus dem am 01.02.2018 in Kraft getretenen Übereinkommen des Europarats zu Verhütung und Bekämpfung von Gewalt gegen Frauen und häuslicher Gewalt, der sog. Istanbul-Konvention. Gemäß Artikel 25 der Istanbul-Konvention sind die Staaten verpflichtet, medizinische und rechtsmedizinische Untersuchungen nach sexualisierter Gewalt sowie Traumahilfen und Beratungen vorzuhalten. Die Einrichtungen müssen für die Betroffenen "leicht zugänglich" und "in ausreichender Zahl" vorhanden sein. Zudem regelt Artikel 15, dass ein „Angebot an geeigneten Aus- und Fortbildungsmaßnahmen zur Verhütung und Aufdeckung" von Gewalt bereitgestellt werden soll [4]. Die Umsetzung dieser Regelungen verläuft in den einzelnen Bundesländern derzeit sehr heterogen. Eine flächendeckende Versorgung mit solchen Einrichtungen, die den erforderlichen medizinisch-forensischen Aspekten gerecht werden und für Betroffene ( $v$. a. auch in ländlichen Bereichen) leicht zugänglich sind, ist in Deutschland bislang nicht gegeben.

Die letzten Ergebnisse zur Erfassung der rechtsmedizinischen Versorgungsstruktur für gewaltbetroffene Personen wurden im Jahr 2012 publiziert [8]. Die Strukturen unterliegen einem stetigen Wandel, sodass durch die Arbeitsgemeinschaft „Klinische Rechtsmedizin“ der Deutschen Gesellschaft für Rechtsmedizin (DGRM) die Ergebnisse einer Umfrage zu aktuellen, an rechtsmedizinischen Instituten in Deutschland angesiedelten Projekten zur Gewaltopferversorgung ausgewertet wurden. Es wurden Inhalte und Strukturen bestehender Versorgungseinrichtungen und Projekte abgefragt, um „Best-practice"-Beispiele zu ermitteln und eine allgemeine Argumentationsgrundlage auf bundesund landespolitischer Ebene vor dem Hintergrund aktueller gesundheitspolitischer Forderungen zu bieten. Auch sollen die Ergebnisse der Erhebung den rechtsmedizinischen Instituten die Möglichkeit bieten, sich bei der Etablierung eigener Versorgungsstrukturen an bereits bestehenden, gut etablierten Modellen zu orientieren.

\section{Methode}

Durch die Arbeitsgemeinschaft Klinische Rechtsmedizin der DGRM wurde ein 4-seitiger Fragebogen zur Erfassung der rechtsmedizinischen Versorgungssituation von Gewaltbetroffenen erarbeitet. Die anschließende Umfrage an rechtsmedizinischen Instituten in Deutschland wurde im Frühsommer 2020 onlinebasiert mittels "SoSci Survey" durchgeführt. Der zugehörige Link zur Umfrage wurde in einem kurzen Anschreiben an die jeweiligen Direktor*Innen der Institute, ggf. zur Weiterleitung an die Projektverantwortlichen, versendet.

Der Fragebogen umfasste insgesamt 20 Fragen. Die Antwortmöglichkeiten wurden - zur besseren Auswertbarkeit - überwiegend in "Multiple-choice"-Form angeboten; bei einigen Fragen waren (zusätzlich) offene Texteingaben vorgesehen.

Es wurden im Wesentlichen folgende Rubriken abgefragt:

- Projekt-/Versorgungsstruktur,

- Leistungsspektrum (Umfang und Art der Untersuchungen, Transport und Lagerung von Asservaten, Fortbildungsangebot),

- beteiligte Akteure und Kooperationen,

- Finanzierung,

- Vor- und Nachteile der Angebote,

- Optimierungsbedarf.

Die Auswertung der erhaltenen Daten, einschließlich Diagrammerstellung, wurde mit Microsoft Office Excel 2019 (Microsoft Corporation, Redmond, WA, USA) durchgeführt.

\section{Ergebnisse}

\section{Allgemeines}

Es wurden insgesamt 16 federführend unter der Leitung der Rechtsmedizin stehende Projekte und Modelle zur Gewaltopferversorgung aus 14 Bundesländern zurückgemeldet. Drei weitere Projekte stehen unter anderer Projektleitung (Frauennotrufe, Arbeitskreis Sexualdelikte); diese wurden nicht in die weitere Auswertung einbezogen. Die Versorgungsgebiete umfassen in 11 Projekten das jeweilige Einzugsgebiet des rechtsmedizinischen Instituts und in 5 Projekten das gesamte Bundesland. 
Tab. 1 Leistungsspektrum der Projekte („multiple choice“)

\begin{tabular}{|l|l|l|}
\hline Leistungsspektrum (Mehrfachantworten möglich) & $n$ & $\begin{array}{l}\text { Prozent der } \\
\text { Fälle (\%) }\end{array}$ \\
\hline $\begin{array}{l}\text { Beratende Funktion } \\
\text { (E-Mail/Telefon) }\end{array}$ & 16 & 100,00 \\
\hline Körperliche Untersuchungen im Institut (Ambulanz für Gewaltopfer) & 15 & 93,75 \\
\hline $\begin{array}{l}\text { Körperliche Untersuchungen konsiliarisch am eigenen Standort } \\
\text { (Klinik der Fakultät) }\end{array}$ & 14 & 87,50 \\
\hline Körperliche Untersuchungen an peripheren Standorten & 13 & 81,25 \\
\hline $\begin{array}{l}\text { Untersuchungen von Erwachsenen } \\
\text { (Körperliche und sexualisierte Gewalt) }\end{array}$ & 14 & 87,50 \\
\hline Untersuchungen bei Kindesmisshandlung & 13 & 81,25 \\
\hline Untersuchungen bei sexuellem Kindesmissbrauch & 12 & 75,00 \\
\hline $\begin{array}{l}\text { Koordinierende Funktion (Kooperationspartner führen Untersuchun- } \\
\text { gen durch) }\end{array}$ & 9 & 56,30 \\
\hline Entwicklung/Betreuung einer Onlineplattform o. Ä. & 5 & 31,25 \\
\hline Einlagerung von (Foto-)Dokumentationen & 16 & 100,00 \\
\hline Einlagerung von Asservaten (Abstriche, Blut-/Urinproben etc.) & 14 & 87,50 \\
\hline Übersendung von Asservaten per Post/Kurier & 11 & 68,75 \\
\hline Fortbildungen/Schulungen regelmäßig bzw. auf Anfrage & 16 & 100,00 \\
\hline
\end{tabular}

\begin{tabular}{|l|l|}
\hline \multicolumn{2}{|l|}{ Tab. 2 Vor- und Nachteile der Projekte (Freitexteingabe) } \\
\hline Vorteile & Nachteile \\
\hline $\begin{array}{l}\text { Niederschwelliges, } \\
\text { kostenfreies Angebot } \\
\text { für die Betroffenen }\end{array}$ & $\begin{array}{l}\text { Unterfinanzierung, Erbringung von unentgeltlichen Leistungen (z. B. } \\
\text { Nacht- und Wochenenddienste, Untersuchungen ohne behördlichen } \\
\text { Auftrag) }\end{array}$ \\
\hline $\begin{array}{l}\text { Wohnortnahe Untersu- } \\
\text { chungen }\end{array}$ & $\begin{array}{l}\text { Keine flächendeckende Versorgung, lange Fahrtwege für Betroffene } \\
\text { und/oder Ärzt*Innen }\end{array}$ \\
\hline Gestiegene Fallzahlen & Personalaufstockung und Verstetigung von Verträgen nicht möglich \\
\hline $\begin{array}{l}\text { Bereitstellen von Be- } \\
\text { funden für ein späteres } \\
\text { Ermittlungsverfahren }\end{array}$ & $\begin{array}{l}\text { Qualität der klinischen Befunddokumentation für ein Strafverfahren } \\
\text { oft unzureichend, lediglich Anforderung von Befundberichten ohne } \\
\text { ausführlichen Gutachtenauftrag (fehlende forensische Interpretation) }\end{array}$ \\
\hline $\begin{array}{l}\text { Interdisziplinärer Aus- } \\
\text { tausch, Entlastung } \\
\text { klinischer Kollegen }\end{array}$ & \begin{tabular}{l} 
Auslagerung rechtsmedizinischer Expertise \\
\hline $\begin{array}{l}\text { Rechtsmedizinischer } \\
\text { Facharztstandard, gute } \\
\text { räumliche Ausstattung }\end{array}$
\end{tabular} \\
\hline $\begin{array}{l}\text { Erzielung von For- } \\
\text { schungsergebnissen/ } \\
\text { Publikationen }\end{array}$ & Zu wenig Anerkennung für die Thematik im eigenen Fach \\
\hline
\end{tabular}

\section{Leistungsspektrum}

Nahezu alle Projekte bieten ein umfassendes Angebot an körperlichen Untersuchungen am eigenen Standort (rechtsmedizinische Ambulanz für Gewaltbetroffene und Klinik der eigenen Fakultät) und in peripheren Einrichtungen (z.B. Kliniken, Praxen, Unterstützungseinrichtungen). Es werden Untersuchungen von Erwachsenen bei körperlicher und sexualisierter Gewalt und in Fällen von Kindesmisshandlung und -missbrauch durchgeführt. Alle Projekte beinhalten zudem ein Bera- tungsangebot per E-Mail/Telefon. In über der Hälfte der Projekte haben die rechtsmedizinischen Institute koordinierende Funktion, wobei die Untersuchungen zusätzlich durch Partnerkliniken durchgeführt werden. In allen Projekten besteht die Möglichkeit der gesicherten Lagerung von (Foto-)Dokumentationen und überwiegend auch von Asservaten wie z.B. Abstrichtupfern, Blut- und Urinproben. Die Dauer der Lagerung variiert und reicht von einem Jahr bis zu 20 Jahren ab Volljährigkeit der betroffenen Person für Asservate und bis zu 25 Jahren für
(Foto-)Dokumentationen. Auch ein regelmäßiges und/oder auf Anfrage verfügbares Schulungs- und Fortbildungsangebot wird in allen Projekten vorgehalten. In einzelnen rechtsmedizinischen Instituten werden elektronische Versorgungsstrukturen geschaffen; diese umfassen neben der Entwicklung von Onlineplattformen auch eine Hilfe-App und ein telemedizinisches Angebot (•Tab. 1).

\section{Beteiligte Akteure}

Abgesehen von der üblichen Zusammenarbeit mit Ermittlungsbehörden/Gerichten und den Finanzgebern (z.B. Ministerien) erfolgt die Kooperation in den rechtsmedizinischen Projekten überwiegend mit Kliniken, Beratungs- und Unterstützungseinrichtungen (z.B. Frauennotrufe, Frauenhäuser, Schutzambulanzen, Traumanetzwerk) sowie Jugendämtern, Einrichtungen der Jugendhilfe und des Kinderschutzes. Selten wurden Kooperationen mit niedergelassenen Arztpraxen, Kinderschutzgruppen sowie der Verein "WEISSER RING" und Anwält*Innen genannt (• Abb. 1 und 2).

\section{Finanzierung}

Zwar wird der Großteil der Projekte über öffentliche Mittel finanziert, jedoch erfolgt die Finanzierung in 7 Projekten ausschließlich oder zusätzlich aus institutseigenen oder sonstigen Mitteln (- Abb. 3). 11 Projekte sind zudem befristet oder Modellprojekte. Die Höhe der Finanzierung aus öffentlichen Mitteln variiert sehr stark und reicht von $25.000 €$ bis $>1.000 .000 €$ jährlich. In 4 Projekten erhalten die Partnerkliniken Aufwandsentschädigungen für Untersuchungen in Form von Fallpauschalen (50-220€/Fall). Unter Berücksichtigung der zurückgemeldeten Projekte lässt sich angeben, dass in Deutschland von der öffentlichen Hand und den rechtsmedizinischen Instituten jährlich mindestens $4.371 .000 €$ für die rechtsmedizinische Versorgung von Betroffenen individueller Gewalt vorgehalten werden.

\section{Vor- und Nachteile der Projekte}

In der Freitexteingabe wurden zu den Vorund Nachteilen der Projekte im Wesentli- 


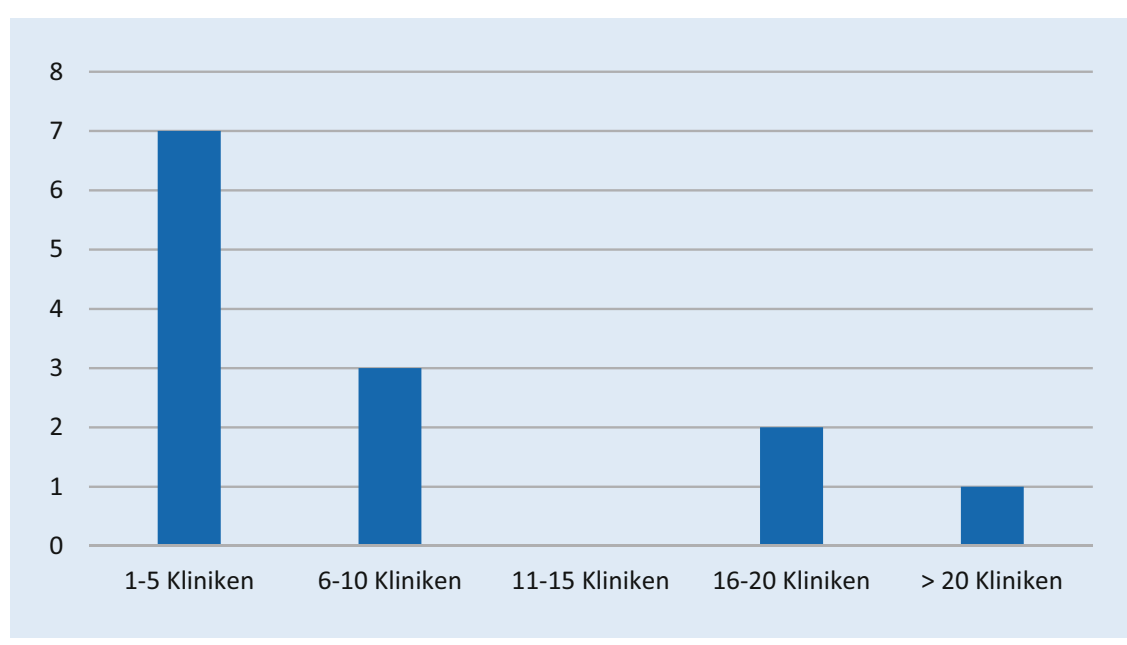

Abb. $1 \Delta$ Anzahl der beteiligten Kliniken in den Projekten (Freitexteingabe)

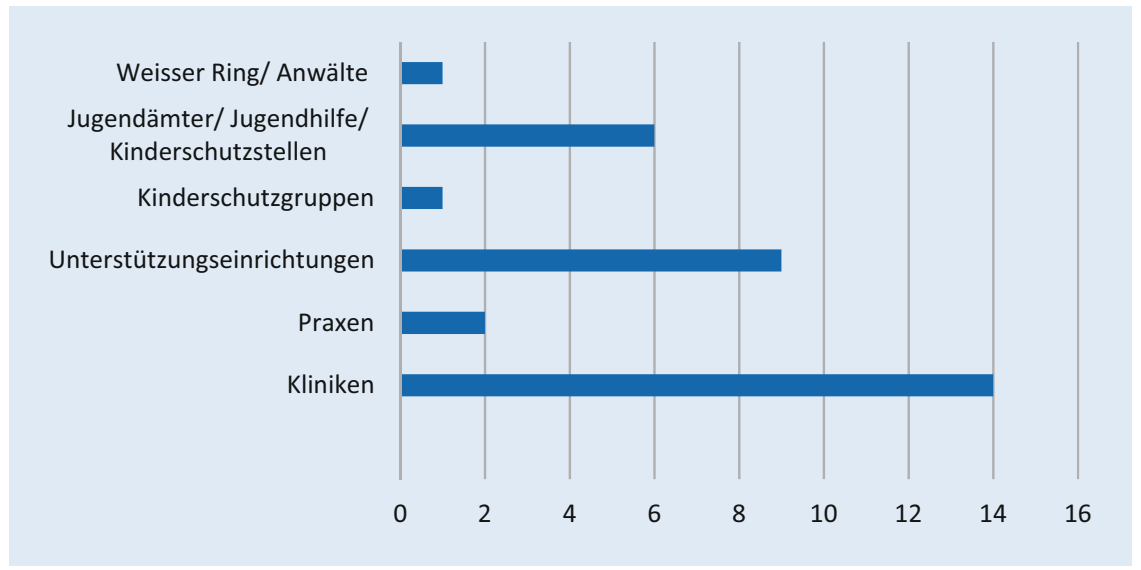

Abb. $2 \Delta$ Beteiligte Akteure in den Projekten, ausgenommen Ermittlungsbehörden, Gerichte und Ministerien (Freitexteingabe)

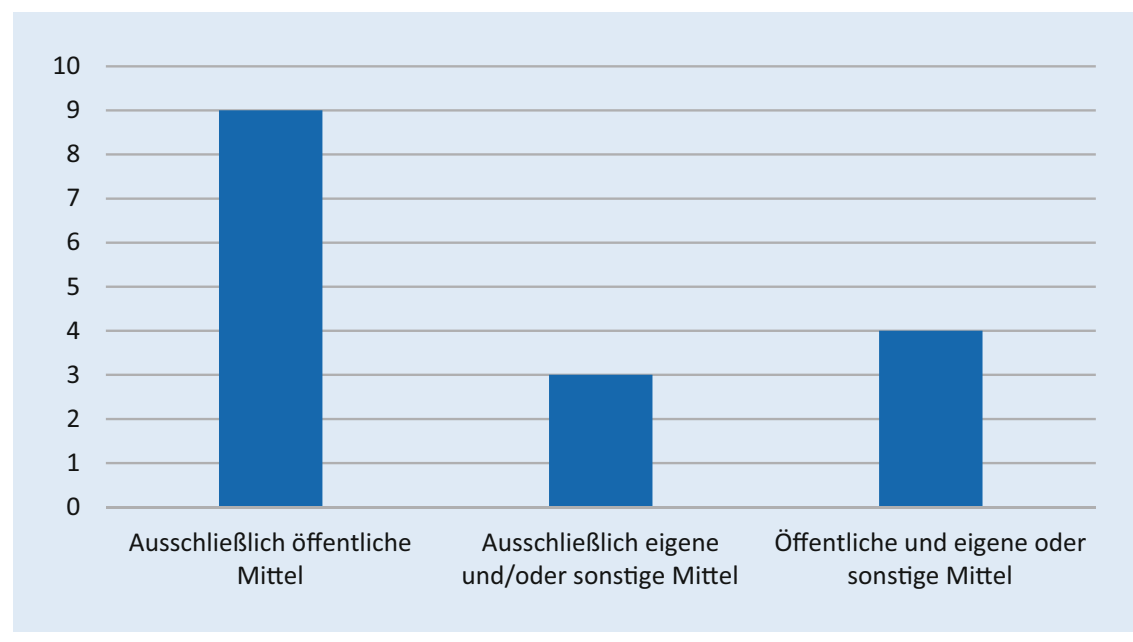

Abb. $3 \Delta$ Finanzierung der Projekte („multiple choice“) chen die in $\mathbf{0}$ Tab. 2 dargestellten Aspekte genannt.

\section{Optimierungsbedarf}

An Optimierungsbedarfen wurden in der Freitexteingabe die Entwicklung und Publikation von verbindlichen Standards für den Einbezug der Rechtsmedizin in die Versorgung von Gewaltbetroffenen und die Erarbeitung von Leitlinien genannt. Als problematisch in diesem Zusammenhang wurde das Vorgehen der Ermittlungsbehörden gesehen, den Betroffenen nach einem Gewaltdelikt lediglich zu empfehlen, „einen Arzt" aufzusuchen, ohne eine qualifizierte Untersuchung in Auftrag zu geben.

Im Falle einer Befunderhebung durch andere Fachdisziplinen (z.B. Gynäkologie bei sexualisierter Gewalt im Rahmen von Kooperationsmodellen) wurde gefordert, die Interpretation der Befunde und Spuren regelhaft durch die Rechtsmedizin vornehmen zu lassen, die über entsprechende Fachkenntnisse verfügt. Darüber hinaus wurde eine Qualitätsprüfung der Befunderhebung durch die Rechtsmedizin als wesentlicher Aspekt genannt. Auch zertifizierte Schulungen für Assistenzärzt*Innen wurden vorgeschlagen, wobei nicht erkennbar war, ob dies auf den Fachbereich der Rechtsmedizin und/oder andere klinische Fachdisziplinen bezogen wurde.

Um die Versorgung in der Fläche zu verbessern, wurden die Schaffung von dezentralen rechtsmedizinischen Anlaufstellen und/oder telemedizinischen Beratungsstrukturen genannt.

Ferner wurde eine gemeinsame Stellungnahme der rechtsmedizinischen Institute (über die DGRM) zur Unterstützung der Institute, die derzeit keine oder nur begrenzt politische Unterstützung erfahren, vorgeschlagen.

Auch die Wahrnehmung der Thematik im Fach Rechtsmedizin und innerhalb der Deutschen Gesellschaft für Rechtsmedizin (DGRM) wurde als optimierungsbedürftig angesehen. Es wurden ein einheitliches Meinungsbild, die Stärkung des rechtsmedizinischen Selbstwertgefühls gegenüber klinischen Kolleg*Innen und eine deutliche Abgrenzung der Rechtsmedizin von anderen Fachdisziplinen gefordert. Insbesondere sollten rechtsmedizinische Aufgaben nicht durch andere Fachgebiete übernom- 
men werden; die Kompetenzen sollten klar getrennt sein.

Letztlich ergab die Auswertung, dass die neuen Bestimmungen zur Finanzierung der vertraulichen Spurensicherung derzeit noch nicht zufriedenstellend umgesetzt sind. Da es sich um Untersuchungen für ein ggf. späteres Strafverfahren handelt, wurde wiederum auf die Beachtung eines rechtsmedizinischen Facharztstandards hingewiesen.

\section{Diskussion}

Die an rechtsmedizinischen Instituten durchgeführte Umfrage ergab ein umfassendes rechtsmedizinisch initiiertes Angebot in unterschiedlicher Organisationsstruktur für die Versorgung von Gewaltbetroffenen in Deutschland. Die Angebote richten sich vorwiegend an Betroffene individueller Gewalt, die meist im privaten Umfeld geschieht. Die Ergebnisse verdeutlichen, dass sich das rechtsmedizinische Leistungsangebot sehr gut in die jeweiligen lokalen Netzwerkstrukturen zur Versorgung von gewaltbetroffenen Personen einbinden lässt. In welchem Umfang anzeige- und verfahrensunabhängige Versorgungsangebote bestehen, ist stark von regionalen (politischen) Regelungen, Projekten und Netzwerkstrukturen abhängig. Untersuchungen von Gewaltbetroffenen können in Flächenbundesländern häufig nicht allein durch die rechtsmedizinischen Institute geleistet werden, die den Universitätskliniken angegliedert sind. In wenigen Bundesländern sind landesweite Versorgungsansätze etabliert. Die Versorgung ist häufig durch rechtsmedizinische Ambulanzen am eigenen Institut und auswärtige rechtsmedizinische Untersuchungen an den eigenen Universitätskliniken und peripheren Standorten geregelt. Zum Teil wurden (zusätzlich) dezentrale Anlaufstellen unter rechtsmedizinischer Federführung geschaffen, wobei die Untersuchungen durch Partnerkliniken erfolgen. Es besteht die Möglichkeit der gesicherten Lagerung von Asservaten und Dokumentationen in rechtsmedizinischen Instituten, was für eine lückenlose Beweiskette in Strafverfahren von großer Bedeutung ist. Daneben verfügen alle Projekte über ein umfassendes Fortbildungsprogramm. Bis auf wenige Ausnah- men werden in den zurückgemeldeten Projekten sowohl Untersuchungen von Kindern als auch von Erwachsenen bei körperlicher und sexualisierter Gewalt abgebildet.

Die Versorgungsgebiete umfassen vorwiegend die Einzugsgebiete der Institute und nur selten das jeweilige gesamte Bundesland. Lange Fahrtwege wurden in den Projekten als nachteilig angesehen. Vor diesem Hintergrund wird deutlich, dass die in der Istanbul-Konvention geforderte flächendeckende und spezialisierte Versorgung von Gewaltbetroffenen derzeit in Deutschland vielerorts nicht gegeben ist.

Nach Angaben einer Expertengruppe für die Bekämpfung von Gewalt gegen Frauen und häusliche Gewalt (Group of Experts on Action against Violence against Women and Domestic Violence, GREVIO) ${ }^{1}$ wird in Anbetracht der Istanbul-Konvention als Richtwert empfohlen, dass pro 200.000 Einwohner*Innen eine Einrichtung, welche Leistungen für die Versorgung von Gewaltbetroffenen erbringt, bereitgestellt wird. Die Angebote sollen räumlich so verteilt sein, dass sie für alle Betroffenen zugänglich sind, unabhängig davon, ob sie in ländlichen Bezirken oder in der Stadt leben [5]. Für RheinlandPfalz würde dies beispielhaft bedeuten, dass ca. 20 spezialisierte Versorgungseinrichtungen geschaffen werden müssten,

\footnotetext{
1 GREVIO ist eine unabhängige Expertengruppe, die für die Überwachung der Umsetzung der Istanbul-Konvention zuständig ist. GREVIO verfasst regelmäßig Berichte über den Stand der Umsetzung der Konvention in den Vertragsstaaten.
}

um den Anforderungen der Konvention gerecht zu werden.

Sowohl die vorliegende als auch die zurückliegende Umfrage zeigen, dass die Rechtsmedizin bereits seit Langem über die Strukturen und die Fachexpertise verfügt, die zum Aufbau einer spezialisierten, flächendeckenden Gewaltopferversorgung erforderlich sind [8]. Seither wurde das Leistungsangebot um Modelle mit Kooperationspartnern (Partnerkliniken) und onlinebasierte Versorgungsstrukturen erweitert, die Finanzierung der Angebote stellt sich jedoch weiterhin problematisch dar [8].

Bei der Bewertung der Nachteile und der Optimierungsvorschläge der Projekte wird deutlich, dass häufig Qualitätsmängel in der ärztlichen, nichtrechtsmedizinischen Befunddokumentation nach Gewaltdelikten vorliegen, was für die juristische Bewertung in späteren Strafverfahren äußerst problematisch ist und zu Retraumatisierungen der Betroffenen führen kann [1, 15-17]. Laut GREVIO können durch die Einbeziehung der Rechtsmedizin in den Ausbau flächendeckender Versorgungsstrukturen unerwünschte Weiterverweisungen und Mehrfachuntersuchungen der Betroffenen als weitere Retraumatisierungsfaktoren vermieden werden [5].

Sowohl der Europarat als auch GREVIO empfehlen, die Versorgung unabhängig von der Erstattung einer Strafanzeige sicherzustellen. Die forensische Untersuchung soll für die Betroffenen kostenlos sein, da die Inanspruchnahme der Strukturen und der Zugang für Angehörige bestimmter Gruppen andernfalls erschwert werden könnten [5]. Diese Möglichkeiten

$$
\begin{aligned}
& \text { Hier steht eine Anzeige. } \\
& \text { Springer }
\end{aligned}
$$


werden auch in den zurückgemeldeten rechtsmedizinischen Modellen als vorteilhaft angesehen, sie können jedoch nur mit einer stetigen Finanzierung angeboten und gewährleistet werden.

Um die in der Istanbul-Konvention genannten Bestrebungen zur Versorgung von Gewaltbetroffenen zu realisieren, sind angesichts der erhobenen aktuellen Versorgungssituation durch rechtsmedizinische Institute spezifische Forderungen zu stellen. Die Finanzierung der Projekte, einschließlich der Deckung des notwendigen Personalbedarfs, ist für die rechtsmedizinischen Institute eine erhebliche Herausforderung, da die erforderlichen Mittel (wenn überhaupt) bundesweit sehr unterschiedlich vergeben werden. Das niederschwellige Untersuchungsangebot bedarf einer auskömmlichen, nichthaushaltsgebundenen Finanzierung, z. B. durch die Aufnahme der rechtsmedizinischen Versorgung in die Gebührenordnung für Ärzte (GOÄ) und/oder Diagnosis Related Groups (DRG). Eine verstetigte Finanzierung, inklusive der Vorhaltekosten (z. B. für Personal), ist notwendig, um die z. T. aus eigenen Mitteln finanzierte Versorgung aufrechtzuerhalten und auszubauen.

Die Ergebnisse der vorliegenden Umfrage bieten eine Argumentationsgrundlage hinsichtlich des Stellenwertes der Rechtsmedizin und einer nachhaltigen Finanzierung rechtsmedizinischer Angebote in der Versorgung von Gewaltbetroffenen. Um eine umfassende politische Unterstützung für die dargestellten Vorhaben zu erhalten, ist eine einheitliche Positionierung im Fach Rechtsmedizin und der DGRM zu der Thematik unerlässlich. In einem offenen Brief der DGRM an den Gesundheitsminister im Oktober 2019 und in einem gemeinsamen Positionspapier der DGRM und des Berufsverbandes Deutscher Rechtsmediziner (BDRM) im April 2020 wurde zur geplanten Kostenübernahme der gesetzlichen Krankenkassen und zur Einbeziehung der Rechtsmedizin in die Umsetzung der Vorgaben im Masernschutzgesetz bereits Stellung genommen [11, 12]. Konkrete Maßnahmen zur Einbeziehung der Rechtsmedizin wurden derzeit noch nicht ergriffen.

Eine flächendeckende Versorgung von Gewaltbetroffenen unter Einhaltung eines gerichtsverwertbaren Untersuchungs- standards stellt eine wesentliche Grundlage zur Umsetzung der Forderungen des Europarates und der Bundesregierung dar. Die angestrebten Ziele in den Beschlüssen der Istanbul-Konvention, im Koalitionsvertrag der Bundesregierung und im Masernschutzgesetz sollten sowohl für Betroffene von körperlicher als auch sexualisierter Gewalt gelten. Die Ergebnisse der Umfrage zeigen, dass rechtsmedizinische Institute über die hierzu erforderliche Expertise und Strukturen verfügen. Grundsätzlich sollten rechtsmedizinische Untersuchungen und Beurteilungen im direkten Kontakt mit den Betroffenen durchgeführt werden. Durch die Schaffung dezentraler Anlaufstellen für Gewaltbetroffene, Schulungen von Kooperationspartnern (z. B. Partnerkliniken) und den Ausbau telemedizinischer Konzepte könnte deutschlandweit unter einem strukturierten Qualitätsmanagement durch die Rechtsmedizin auch in der Peripherie ein forensischer Standard von Untersuchungen zur lückenlosen Beweissicherung etabliert werden.

\section{Fazit}

Rechtsmedizinische Institute bieten in nahezu allen Bundesländern ein umfassendes niederschwelliges Angebot auf dem erforderlichen fachlichen Qualitätsniveau. Die Finanzierungsmodelle sind allerdings sehr heterogen, häufig befristet und nicht auskömmlich. Die rechtsmedizinische Expertise wird häufig leichtfertig ausgelagert, wodurch ein gerichtsverwertbarer Standard oft nicht gegeben ist. Um die Versorgung von Gewaltbetroffenen entsprechend den neuen Bestimmungen des Bundestages langfristig auf hohem Niveau zu gewährleisten, sind eine bundesweite Stärkung der Rolle der Rechtsmedizin in der Versorgung von Gewaltbetroffenen, politische Unterstützung sowie auskömmliche Finanzierung dringend zu fordern.

\section{Korrespondenzadresse}

\section{Dr. med. Cleo Walz}

Institut für Rechtsmedizin, Universitätsmedizin der Johannes Gutenberg-Universität Mainz Am Pulverturm 3, 55131 Mainz, Deutschland walz@uni-mainz.de
Danksagung. Die Autorinnen danken der Arbeitsgemeinschaft Klinische Rechtsmedizin, welche die Gestaltung der Umfrage maßgeblich unterstützt hat.

Funding. Open Access funding enabled and organized by Projekt DEAL.

\section{Einhaltung ethischer Richtlinien}

Interessenkonflikt. C. Walz, N. Wilke-Schalhorst, C.-S. Schwarz und T. Germerott geben an, dass kein Interessenkonflikt besteht.

Für diesen Beitrag wurden von den Autoren keine Studien an Menschen oder Tieren durchgeführt. Für die aufgeführten Studien gelten die jeweils dort angegebenen ethischen Richtlinien.

Open Access. Dieser Artikel wird unter der Creative Commons Namensnennung 4.0 International Lizenz veröffentlicht, welche die Nutzung, Vervielfältigung, Bearbeitung, Verbreitung und Wiedergabe in jeglichem Medium und Format erlaubt, sofern Sie den/die ursprünglichen Autor(en) und die Quelle ordnungsgemäß nennen, einen Link zur Creative Commons Lizenz beifügen und angeben, ob Änderungen vorgenommen wurden.

Die in diesem Artikel enthaltenen Bilder und sonstiges Drittmaterial unterliegen ebenfalls der genannten Creative Commons Lizenz, sofern sich aus der Abbildungslegende nichts anderes ergibt. Sofern das betreffende Material nicht unter der genannten Creative Commons Lizenz steht und die betreffende Handlung nicht nach gesetzlichen Vorschriften erlaubt ist, ist für die oben aufgeführten Weiterverwendungen des Materials die Einwilligung des jeweiligen Rechteinhabers einzuholen.

Weitere Details zur Lizenz entnehmen Sie bitte der Lizenzinformation auf http://creativecommons.org/ licenses/by/4.0/deed.de.

\section{Literatur}

1. Bode-Jänisch S, Meyer Y, Schroeder G et al (2011) Clinical forensic examination findings and legal outcome in cases of suspected physical child abuse. Arch Kriminol 228:73-81

2. Brackrock D, Dokter M, Eckhoff C et al (2020) Gewalt gegen Frauen - eine Auswertung von sieben Jahren Gewaltopferambulanz am Institut für Rechtsmedizin Greifswald (2011-2017). Rechtsmedizin 30:153-160

3. Bundesgesetzblatt Jahrgang 2020 Teil I Nr. 6. Gesetz für den Schutz vor Masern und zur Stärkung der Impfprävention (Masernschutzgesetz).

4. Council of Europe Treaty Series (2011) Übereinkommen des Europarats zur Verhütung und Bekämpfung von Gewalt gegen Frauen und häuslicher Gewalt und erläuternder Bericht. https://rm coe.int/1680462535.Zugegriffen: 17. Febr. 2021

5. Fischer $L$ (2020) Akutversorgung nach sexualisierter Gewalt. Zur Umsetzung von Artikel 25 der Istanbul-Konvention in Deutschland. https://www. institut-fuer-menschenrechte.de/fileadmin/ Redaktion/Publikationen/Analyse_Studie/ Analyse_Akutversorgung_nach_sexualisierter Gewalt.pdf.Zugegriffen: 23. Febr. 2021 (Deutsches Institutfür Menschenrechte) 
6. Koalitionsvertrag 2021-2025 zwischen SPD, BÜNDNIS 90/DIE GRÜNEN und FDP. Mehr Fortschritt wagen. Bündnis für Freiheit, Gerechtigkeit und Nachhaltigkeit. Schutz vor Gewalt: 114.

7. Koalitionsvertrag 2021-2025 zwischen SPD, BÜNDNIS 90/DIE GRÜNEN und FDP. Mehr Fortschritt wagen. Bündnis für Freiheit, Gerechtigkeit und Nachhaltigkeit. Schutz vor Gewalt: 115.

8. Gahr B, Graß H, Ritz-Timme S, Banaschak S (2012) Klinisch-rechtsmedizinische Kompetenz in der Gewaltopferversorgung. Was leisten Institute für Rechtsmedizin in Deutschland, Österreich und der Schweiz? Rechtsmedizin 22:379-384

9. Graß H, Ritz-Timme S (2005) Rechtsmedizinische Kompetenz im Netzwerk gegen häusliche Gewalt. JNetzw Frauenforsch NRW 19:49-51

10. Graß H, Rothschild MA (2004) Klinische Rechtsmedizin. Aufgaben und Herausforderungen im Rahmen der medizinischen Betreuung von Opfern häuslicher Gewalt. Rechtsmedizin 14:188-192

11. https://www.dgrm.de/fileadmin/PDF/PDF_ Duesseldorf/Stellungnahme_Umsetzung_des_ Masernschutzgesetzes_zur_Vertraulichen_ Spurensicherung_Einbeziehung_der Rechtsmedizin.pdf. Zugegriffen: 23. Febr. 2021

12. https://www.dgrm.de/fileadmin/PDF/PDF_ Muenchen/Brief_Spahn.2019.pdf. Zugegriffen: 23. Febr. 2021

13. Jundt K, Friese K (2009) Gewalt an Frauen. Gynäkologe 42:723-727

14. Jungbluth P, Grassmann JP, Wild M et al (2012) Gewaltopferversorgung in der Notfallambulanz. Rechtsmedizin 22:163-168

15. McGregor MJ, Du Mont J, Myhr TL (2002) Sexual assault forensic medical examination: Is evidence related to successful prosecution? Ann Emerg Med 39:639-647

16. Rees G (2011) Morphology is a witness which doesn't lie: diagnosis by similarity relation and analogical inference in clinical forensic medicine. Soc Sci Med 73:866-872

17. Wyler D, Gerlach K, Klopfstein U et al (2005) Examination of victims of sexual violence-the problem offalse reports. Ther Umsch 62:255-259

\section{Forensic models of care for persons affected by violence in Germany}

Background: In Germany, there are no uniform structures for the care of victims of violence. According to current political efforts it should be possible to obtain evidence for violent crimes throughout Germany without having to file criminal charges. Forensic medical institutes have supply structures in order to offer a low-threshold collection of findings that can be used in court.

Material and methods: The Working Group of Clinical Forensic Medicine of the German Society of Forensic Medicine conducted a survey of forensic medical institutes in Germany on current models of care for victims of violence. The survey included information on project structure, range of services and service area, financing and advantages and disadvantages of the projects.

Results: A total of 16 reported projects from 14 states offer physical examinations at their own and peripheral sites, consultations, storage of evidence, and continuing education. In about half of the projects, the institutes of forensic medicine have an additional coordinating function with examinations performed by cooperation partners. The majority of the projects are financed by public funds, in 7 projects own or other funds have to be raised additionally and 11 projects are temporary or model projects.

Discussion: Forensic medical institutes have the necessary expertise and offer a comprehensive low-threshold service for victims of violence. The financing models are heterogeneous, often limited in time and inadequate. In order to establish the politically demanded comprehensive care, a nationwide inclusion of forensic medicine and adequate funding is urgently required.

\section{Keywords}

Clinical forensic medicine · Funding · Outpatient clinics · Istanbul Convention · Measles Protection Act 